\title{
Cooperative communications when collaborators are not synchronized
}

\begin{abstract}
A basic challenge for future wireless communication systems is the contradiction between the fast growing demand for high speed wireless communications and the limited electromagnetic wave frequency spectrum. The multiple-input multiple-output (MIMO) technique with space-time coding (STC) is recognized as a promising scheme for the next-generation of high speed wireless communication systems. This is mainly because of its capability of providing spatial diversity to combat fading in wireless channels and increasing the capacity without additional power consumption or bandwidth expansion. However, all these improvements come at the cost of multiple radio frequency (RF) front ends at both the transmitter and the receiver. The size of the mobile devices and the requirements for distance between antennas may
\end{abstract} limit the number of antennas that can be deployed. The exploitation of the spatial benefits for wireless terminals with only single antennas becomes an obvious question. Cooperative communications technology may provide an answer to this question.

In cooperative communications, as shown in Figure 1, multiple terminals in a wireless network are capable of working together: one terminal can help others transmit their desired signals. As such, the spatial links of both the transmitter and the helper can be exploited and as a result, multiple singleantenna terminals can share their antennas to create a virtual antenna array. Meanwhile, because of their distributed nature, independently fading channels could be guaranteed to provide a spatial degree-of-freedom. Through cooperation it becomes possible to exploit the spatial resource of the traditional MIMO techniques without the need to equip each terminal with multiple antennas. Therefore, it can be considered as a distributed MIMO system, while the tradi-

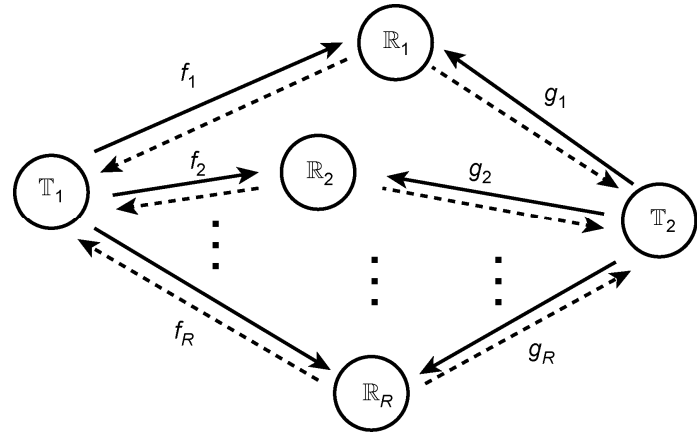

Figure 1 A cooperative communication system. tional MIMO system can be termed a co-located MIMO system. Since the early studies by Sendonaris et al. and Laneman et al., cooperative communications techniques have attracted tremendous interest over recent years, both in academia and industry. Distributed space-time coding (DSTC), i.e. the distributed counterpart of space-time coding in co-located MIMO systems, has become an efficient technique to provide spatial diversity for cooperative communication systems. On the one hand, cooperation is embedded into cellular mobile communications systems and new cooperative stations may be built in a cellular system; on the other hand, a cooperative communications technique is adopted in the physical layer of ad hoc networks and wireless sensor networks (WSN). Compared with the co-located MIMO systems, the most distinctive characteristic of cooperative communications systems is their distributed nature: multiple terminals in cooperation are spatially separated in several different physical locations. Thus, some new problems that do not exist in co-located MIMO systems have emerged: for example, (1) Signal coordination: Whether and when a terminal participates in the cooperation, and what a terminal should transmit. (2) Time asynchronism: Multiple cooperative terminals have different distances to the destination terminal and thus the cooperative transmission may be time-asynchronous. (3) Frequency asynchronism: Each cooperative terminal has its own oscillator and thus the cooperative transmission is frequency-asynchronous.

Although centralized high layer control protocols can be used to solve some of these problems; for example, a central station can be set to guarantee the signal coordination and synchronize the cooperative terminals. However a large number of extra control signals are required, which may greatly reduce the benefits of cooperation. On the other hand, it may be difficult for wireless networks without infrastructure, like an ad hoc network or a WSN, to have a central control station. Also, these networks generally have high dynamics, where nodes frequently join and leave the network, which further significantly increases the overhead. Therefore, researchers take great interest in dealing with these problems using physical layer techniques, such as coding/modulation and advanced signal processing.

An overview of some of the research efforts on this topic in recent years is given in the published paper below, by considering the last two problems, i.e. time and frequency asynchronism. The paper summarizes various distributed STCs and equalization methods that have been proposed for cooperative nodes and destination nodes to combat time and frequency asynchronism, all aimed at achieving as much diversity gain as possible.

See the article: Wang H M, Xia X G. Asynchronous cooperative communication systems: A survey of signal designs. Sci China Inf Sci, 2011, 54: $1547-1561$

Open Access This article is distributed under the terms of the Creative Commons Attribution License which permits any use, distribution, and reproduction in any medium, provided the original author(s) and source are credited. 